\title{
I Informe del Registro Español de Tumores Postrasplante Cardiaco
}

\author{
María G. Crespo-Leiro,, Luis Alonso-Pulpón, Juan F. Delgado Jiménez, Sonia Mirabet, \\ Iago Sousa Casasnovas, Luis Almenar Bonet, Francisco Gonzále-Vílchez, Nicolás \\ Manito Lorite, Beatriz Díaz Molina, Gregorio Rábago
}

\begin{abstract}
Resumen
Las neoplasias son una complicación frecuente y grave tras el trasplante cardiaco y una de las causas más importantes de muerte a largo plazo. El Registro Español de Tumores Postrasplante Cardiaco se inició en 2004, es online e incluye a todos los pacientes con trasplante cardiaco en España y con actualización continua de todos los tumores postrasplante. Los más frecuentes son cutáneos (54\%), seguidos de los tumores no cutáneos no linfoides (39\%) y linfomas (7\%). La incidencia aumenta con la edad y el tiempo postrasplante y es mayor en varones. A los 15 años solo un $62 \%$ de los pacientes están libres de tumores. El pronóstico varía según el tipo de tumor. La incidencia de linfomas ha disminuido a la mitad en la última década. El Registro ayuda a conocer la incidencia, los factores de riesgo y el pronóstico de los tumores postrasplante y establecer estrategias de mejora.
\end{abstract}

Palabras clave

Trasplante cardiaco. Tumores. Complicaciones.

\section{Introducción}

Los avances en la inmunosupresión, que han conseguido mejorar la supervivencia a largo plazo de los pacientes con trasplante, tienen como contrapartida el desarrollo de otras complicaciones; una de ellas es las neoplasias. En la actualidad, las neoplasias son una causa importante de muerte tras el trasplante cardiaco (TxC) y, de hecho, compite con la enfermedad vascular del injerto (o rechazo crónico) como una de las más frecuentes causas de muerte a largo plazo ${ }^{1,2}$. A pesar de la trascendencia de esta entidad, hay muchos aspectos todavía desconocidos y que hacen necesario conocerla en detalle para poder reducir su incidencia o gravedad, aspectos clave para mejorar los resultados a largo plazo del TxC.

La epidemiología de las neoplasias post-TxC es un tema de enorme interés, pero difícil de estudiar. Israel Penn fue el primero en reconocer el aumento en la incidencia de neoplasias tras el trasplante en $1967^{3}$ e inició el registro que lleva su nombre. Ese registro tuvo varias denominaciones. Inicialmente Denver Transplant Tumor Registry, luego Cincinnati Transplant Tumour Registry (CTTR) y, últimamente (desde la muerte de Penn), Israel Penn Internacional Transplant Tumour Registry (IPITTR), disponible online. Pese a que es el mayor registro de neoplasias postrasplante y que proporciona información sobre su tipo y su pronóstico ${ }^{4,5}$, tiene algunas limitaciones. Entre ellas, que se trata de un registro voluntario y que solo recoge datos de pacientes con neoplasia, por lo que no aporta datos de incidencia y no permite identificar factores de riesgo o predisponentes.

En el pasado, una de las principales dificultades para conocer la incidencia de neoplasias post-TxC era que la mayoría de los estudios tenían un pequeño tamaño muestral (por lo general se realizaban en un solo centro), seguimiento corto y, además, solían expresar los resultados en porcentajes ${ }^{6,7,8}$. Puesto que las neoplasias son complicaciones fundamentalmente a largo plazo cuya incidencia generalmente aumenta con el tiempo de seguimiento, la información disponible era escasa y hacía difícil la comparación entre diferentes trabajos. En este sentido, los datos de incidencia deberían expresarse en tasa de incidencia, es decir, en número de neoplasias por unidad de tiempo en relación con el tamaño de la población en riesgo (p. ej., 1.000 pacientes/año de seguimiento). Con la idea de mejorar la información existente en neoplasias post-TxC, nace el Registro Español de Tumores Postrasplante Cardiaco (RETPTC) en el año 2004.

La idea del RETPTC se inició a finales de 2003 como iniciativa de la Sección de Insuficiencia Cardiaca y Trasplante Cardiaco de la Sociedad Española de Cardiología, y en ella participan todos los centros de TxC de España. El objetivo principal es conocer la incidencia, los factores de riesgo y la 
historia natural de las neoplasias post-TxC en España y servir de base para mejorar el conocimiento de esta entidad e identificar áreas de mejora. Entre las características diferenciales con estudios previos, se trata de un registro enteramente online (que facilita la entrada de datos) que incluye información de todos los pacientes con TxC de España, es decir, desde que se inició esta terapia en nuestro país en 1984. El registro incluye, además de las variables epidemiológicas habituales, variables relacionadas específicamente con las neoplasias, como factores de riesgo de tumores previos al TxC, inmunosupresión basal y cambios posteriores, datos de infecciones, empleo de antivirales, datos del tumor codificados según el SNOMED en cuanto a localización y patología, estadio al diagnóstico, tratamiento del tumor y evolución, tanto del tumor como supervivencia. Una mejora adicional tuvo lugar en 2003, al fusionarse este registro con el Registro Español de Trasplante Cardiaco, una vez que este se transformó en registro online. A continuación se presentan los datos del I Informe del RETPTC (1984-2013) y aportaciones científicas relevantes derivadas de este registro en años previos.

\section{I informe del registro español de tumores postrasplante cardiaco (1984-2013)}

En el I Informe del RETPTC (realizado en 2014), se incluyó a 5.172 pacientes, población compuesta por todos los TxC realizados entre 1984 y diciembre de 2013 a pacientes con edad $\geq 16$ años, excluidos los retrasplantes y trasplantes combinados y los pacientes fallecidos en los primeros 3 meses post-TxC. Hubo un total de 1.674 tumores en 1.134 pacientes distintos. La Figura 1 muestra el número acumulado de trasplantes (sin eliminar los fallecidos en los primeros 3 meses), el número de pacientes vivos al final de cada año y el número acumulado de tumores diagnosticados. Se aprecia que el número de tumores postTxC aumenta con el tiempo post-TxC. La Figura 2 muestra la incidencia según el tipo de tumor; los tumores cutáneos son los más frecuentes (54\%), seguidos de tumores no cutáneos no linfoides (39\%) y los linfomas (7\%). La Figura 3 muestra la incidencia de tumores en general y de cada tipo de tumor, expresada en tanto/1.000 personasaño de seguimiento. La Figura 4 muestra la supervivencia libre de tumores, global y por tipo de tumor. A los 15 años, solo un $62 \%$ de los pacientes estaban libres de tumores. La Figura 5 muestra la incidencia de tumores según el régimen inmunosupresor inicial, y se aprecia que la combinación de tacrolimus con micofenolato mofetilo (MMF) es la que tiene menor incidencia de tumores, seguida por la combinación de ciclosporina con MMF y la de ciclosporina con azatioprina. La Figura 6 muestra la incidencia de tumores por década; se aprecia en la última década una reducción en la incidencia respecto a la década previa. Las Figura 7, Figura 8, Figura 9 muestran la incidencia por década según cada tipo de tumor. La Figura 10muestra la incidencia por sexos; se aprecia menor incidencia en las mujeres.

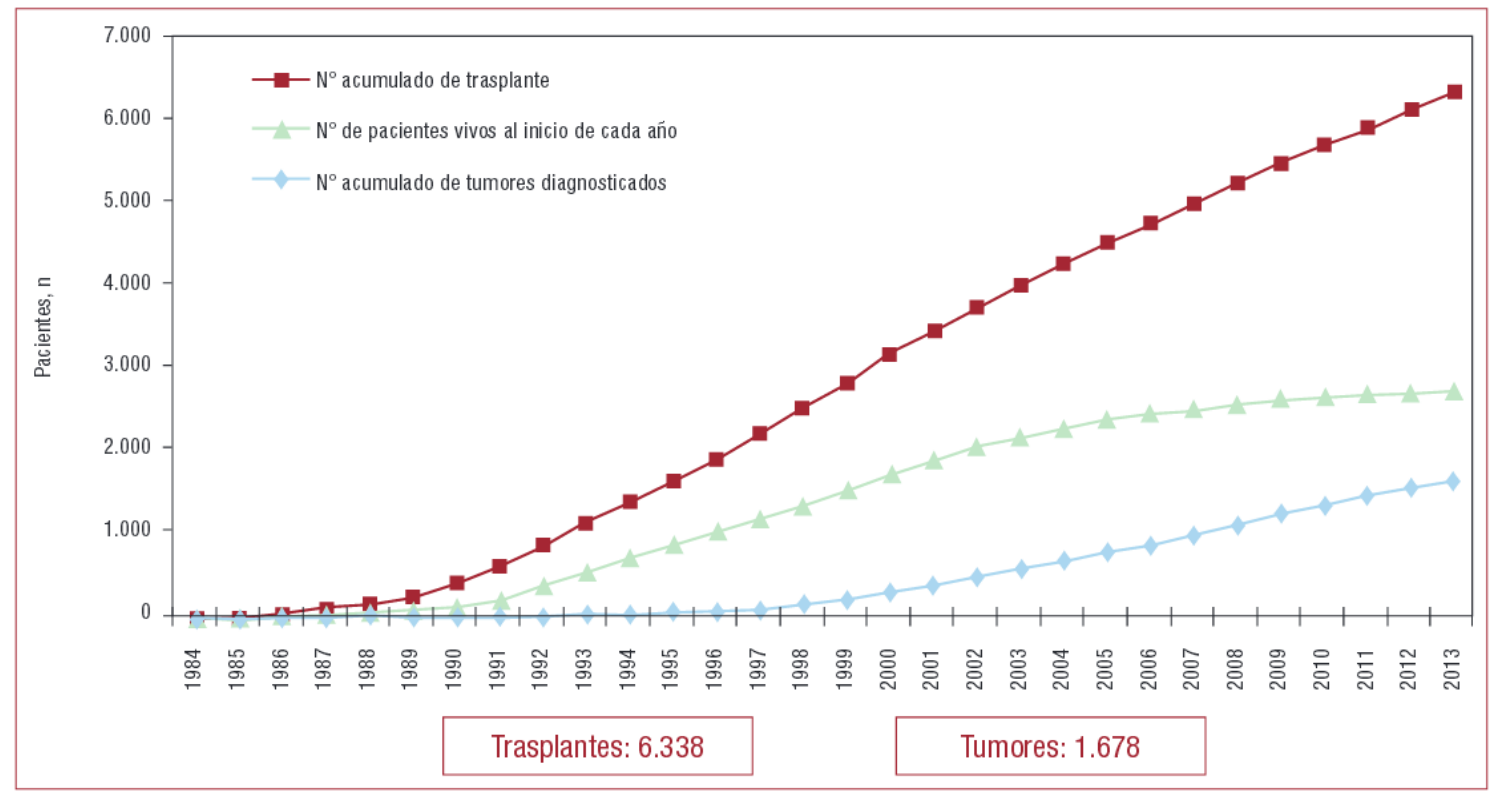

Figura 1. Número de trasplantes y tumores acumulados, sin eliminar a los pacientes fallecidos en los primeros 3 meses. 


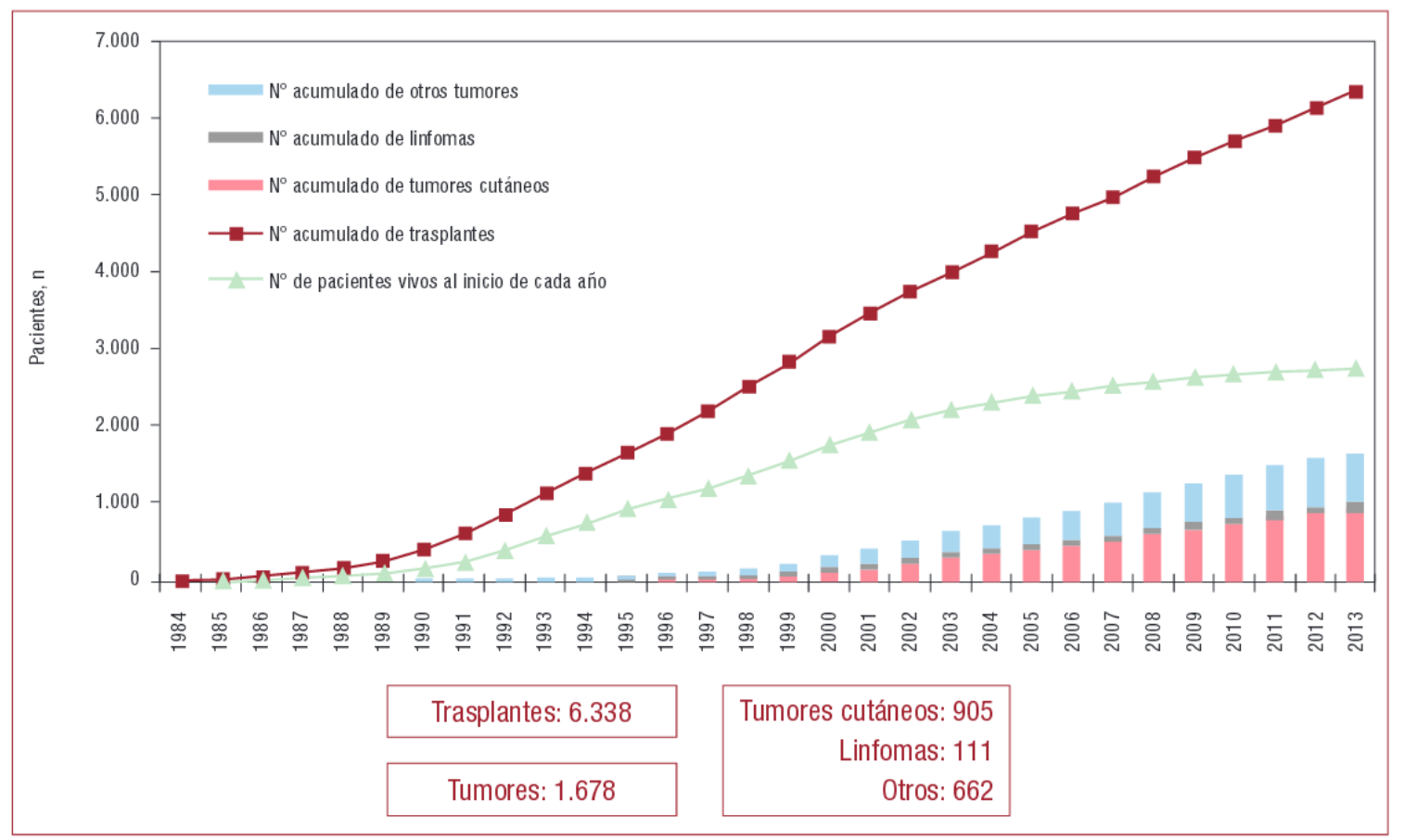

Figura 2. Número de trasplantes y tipo de tumores acumulados, sin eliminar a los pacientes fallecidos en los primeros 3 meses.

\begin{tabular}{|c|c|c|c|c|c|c|}
\hline Tipo de tumor & Tiempo & Personas-an̂o & $\mathrm{N}^{0}$ de tumores & Tasa de incidencia & \multicolumn{2}{|c|}{ IC95\% } \\
\hline \multirow{4}{*}{ Todos } & 1 año & $4.898,0$ & 89 & 18,2 & 14,8 & 22,4 \\
\hline & 5 años & $20.753,0$ & 518 & 25,0 & 22,9 & 27,2 \\
\hline & 10 años & $33.892,4$ & 1.154 & 34,0 & 32,1 & 36,0 \\
\hline & Total & $43.695,6$ & 1.674 & 38,3 & 36,5 & 40,2 \\
\hline \multirow{4}{*}{ Cutáneos } & 1 año & $4.898,0$ & 27 & 5,5 & 3,8 & 8,0 \\
\hline & 5 años & $20.753,0$ & 212 & 10,2 & 8,9 & 11,7 \\
\hline & 10 años & $33.982,4$ & 580 & 17,1 & 15,7 & 18,5 \\
\hline & Total & $43.695,6$ & 904 & 20,7 & 19,4 & 22,1 \\
\hline \multirow{4}{*}{ Linfomas } & 1 año & $4.898,0$ & 16 & 3,3 & 2,0 & 5,3 \\
\hline & 5 años & $20.753,0$ & 49 & 2,4 & 1,8 & 3,1 \\
\hline & 10 años & $33.982,4$ & 83 & 2,4 & 2,0 & 3,0 \\
\hline & Total & $43.695,6$ & 110 & 2,5 & 2,1 & 3,0 \\
\hline \multirow{4}{*}{ Otros } & 1 año & $4.898,0$ & 46 & 9,4 & 7,0 & 12,5 \\
\hline & 5 años & $20.753,0$ & 253 & 12,2 & 10,8 & 13,8 \\
\hline & 10 años & $33.982,4$ & 480 & 14,1 & 12,9 & 15,5 \\
\hline & Total & $43.695,6$ & 647 & 14,8 & 13,7 & 16,0 \\
\hline
\end{tabular}

Figura 3. Incidencia de tumores (tasa cada 1.000 personas-año). IC: intervalo de confianza. 


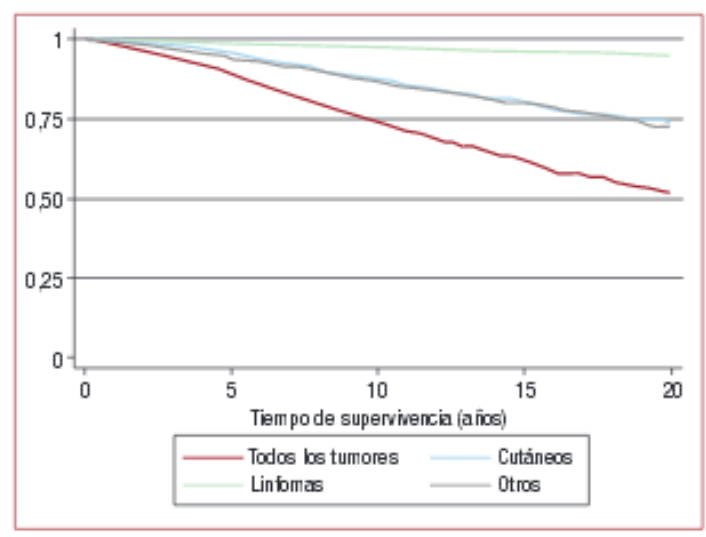

Figura 4. Supervivencia libre de tumores, por tipo.

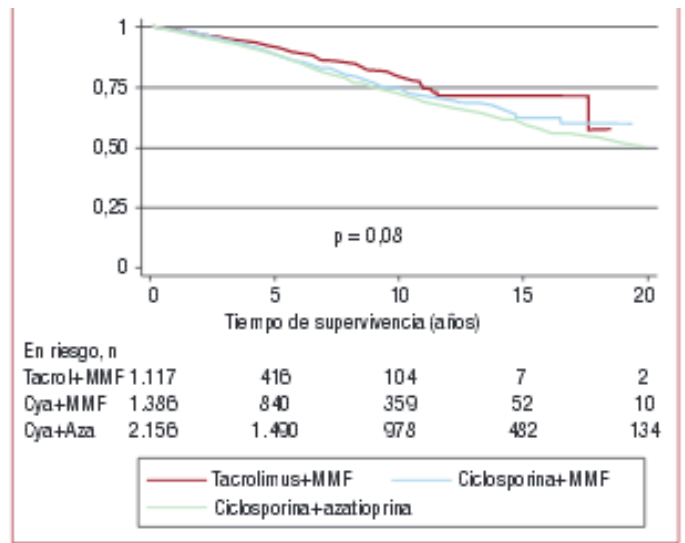

Figura 6. Supervivencia libre de tumores, por década.

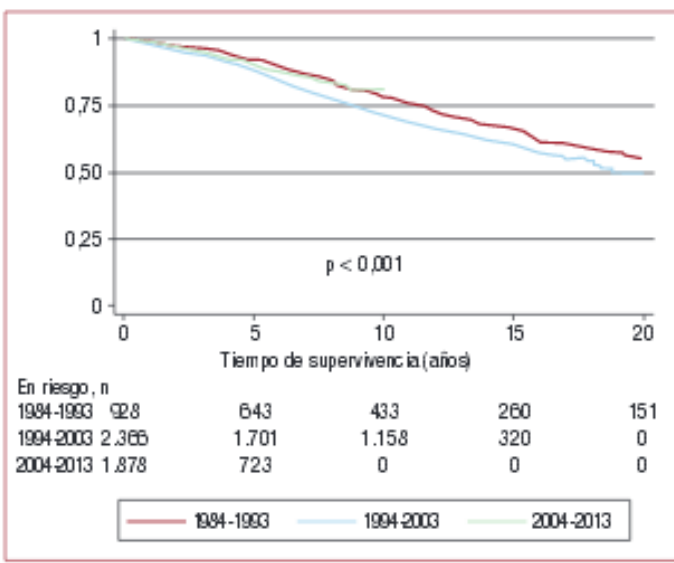

Figura 8. Supervivencia libre de linfomas, por década.

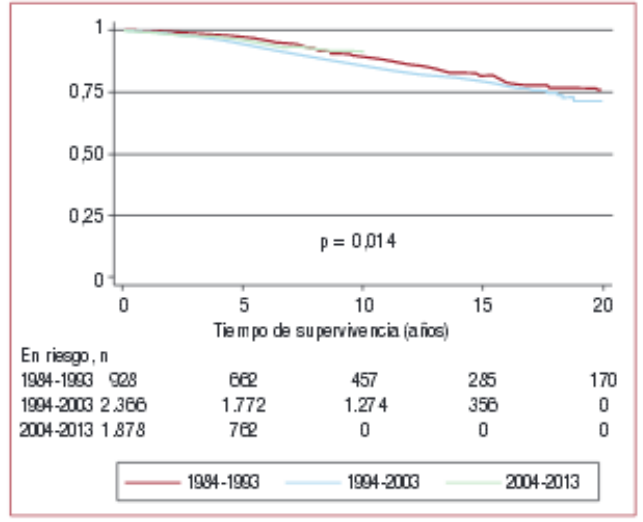

Figura 5. Supervivencia libre de tumores, por inmunosupresión inicial. Aza: azatioprina; Cya: ciclosporina; MMF: micofenolato mofetilo; Tacrol: tacrolimus.

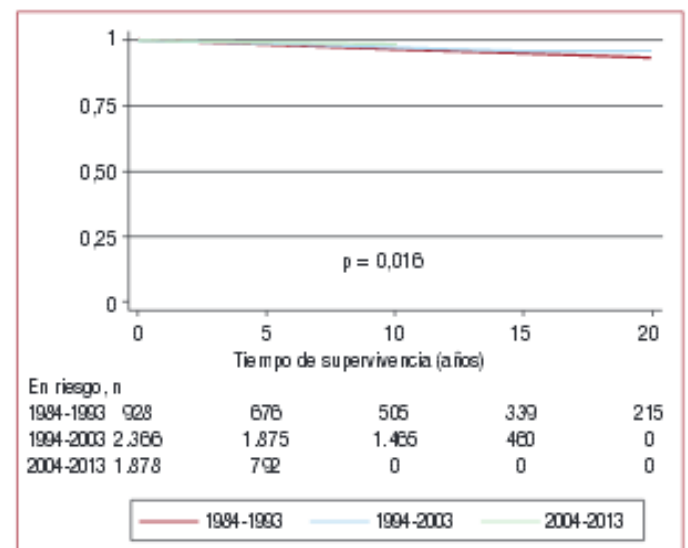

Figura 7. Supervivencia libre de tumores cutáneos, por década.

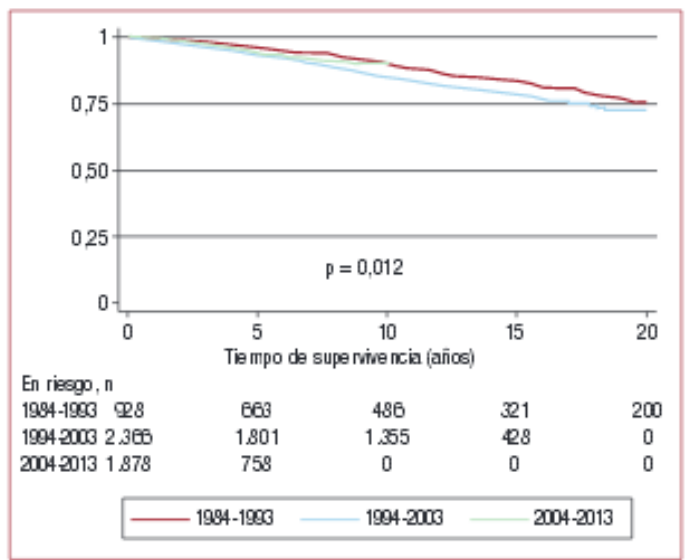

Figura 9. Supervivencia libre de otros tumores, por década. 


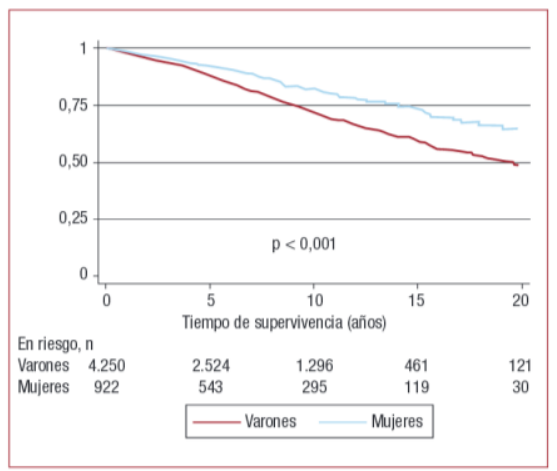

Figura 10. Supervivencia libre de tumores, por sexo.

En cuanto a la supervivencia tras el diagnóstico de un tumor, la Figura 11 muestra la probabilidad de sobrevivir al primer tumor; al año es del $76 \%$ y a los 15 años, del 22\%. La Figura 12 compara la supervivencia al tumor según tipo de tumor; se observa peor supervivencia con linfomas y otros tumores que con los tumores cutáneos. Las Figura 13 y Figura 14Fmuestran la supervivencia al primer tumor diagnosticado en los primeros 3 años (global y según tipo de tumor) comparada con la de los pacientes sin tumor. Se aprecia que la supervivencia a los tumores cutáneos es similar a la de los pacientes sin tumor y significativamente superior a la de los pacientes con linfomas u otros tipos de tumores.

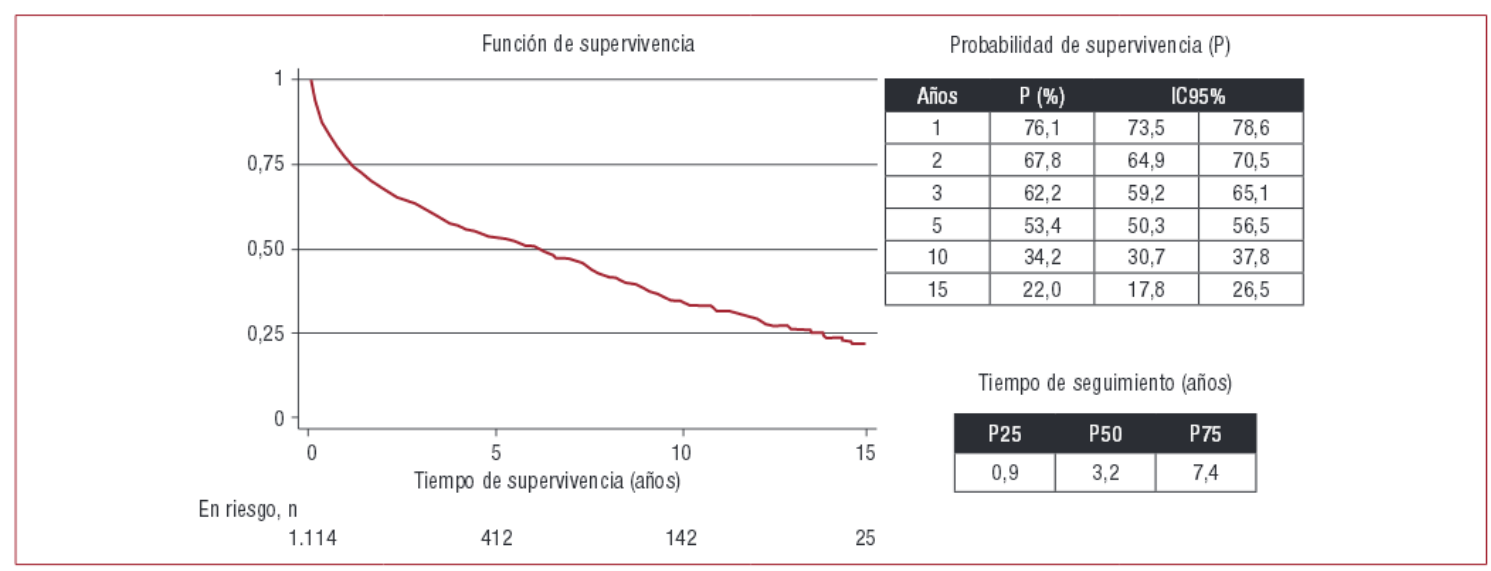

Figura 11. Supervivencia al primer tumor. IC: intervalo de confianza. 


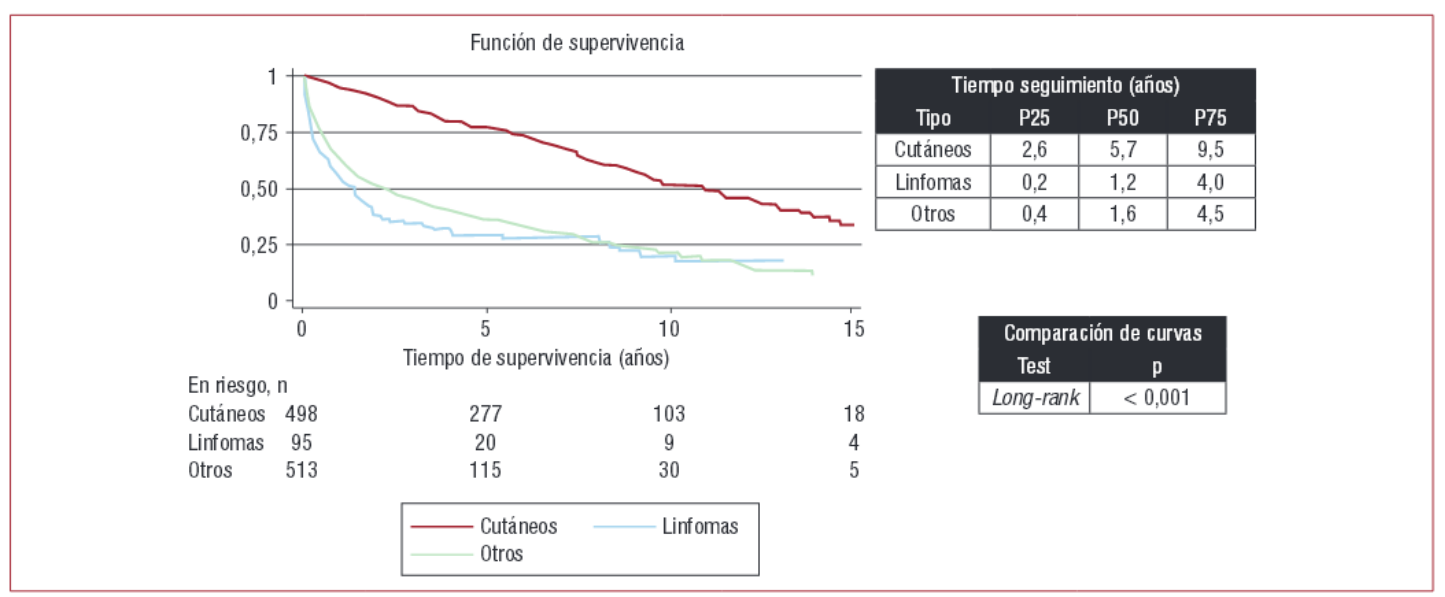

Figura 12. Supervivencia al primer tumor, por tipo.

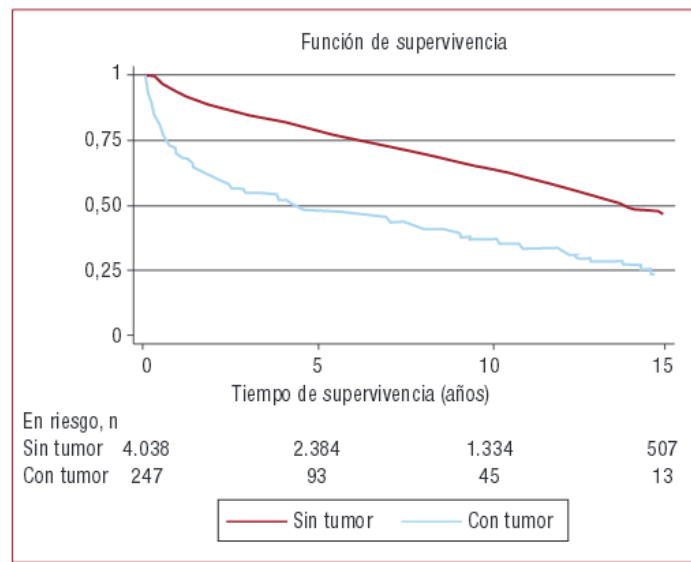

Figura 13. Supervivencia al primer tumor diagnosticado en los primeros 3 años y supervivencia de pacientes sin tumor.

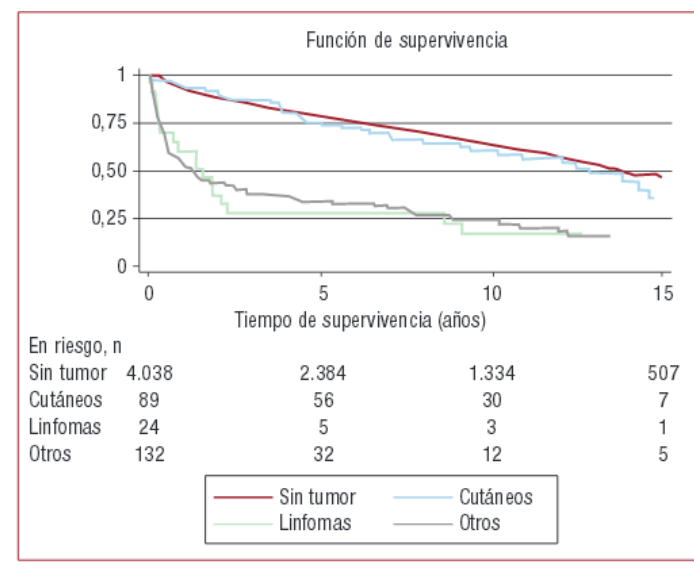

Figura 14. Supervivencia al primer tumor diagnosticado en los primeros 3 años y supervivenciade pacientes sin tumor

\section{Incidencia, factores de riesgo y pronóstico de las neoplasias postrasplante cardiaco}

Uno de los primeros análisis de este Registro ${ }^{9}$ evaluó la incidencia, los factores de riesgo y el pronóstico de las neoplasias post-TxC. Se incluyó a 3.393 pacientes adultos con TxC entre 1984 y 2003, seguidos hasta diciembre de 2004 con una mediana de seguimiento de 5,2 (media, 5,8; máximo, 20,2) años. Hubo 639 neoplasias en 490 pacientes. Un 50\% eran neoplasias cutáneas; el 10\%, linfomas y el 40\%, otros tumores. La incidencia acumulada de tumores cutáneos y otros tumores no linfoides aumentaba con la edad en el momento del TxC y con el tiempo post-TxC (desde 5,2 y 5,8/1.000 personas-año respectivamente en el primer año hasta 14,8 y 12,6 tras 10 años) y era mayor en varones que en mujeres. Sin embargo, ninguna de esas tendencias se observó con los linfomas. El uso de MMF como inmunosupresor basal redujo de forma independiente el riesgo de tumores cutáneos. La supervivencia a los 5 años tras un primer tumor fue del $74 \%$ a tumores cutáneos, el $20 \%$ a linfomas y el $32 \%$ a los otros tumores.

En otra cohorte del RETPTC con mayor tamaño muestral, en la que se incluyó a pacientes con TxC realizado entre 1984 y diciembre de 2008 ( $\mathrm{n}=4.359)$, se analizaron los 375 tumores no cutáneos y no linfoides, es decir, los que denominamos «otros» tumores ${ }^{10}$. Las localizaciones más frecuentes fueron pulmón $(25,9 \%)$, tracto gastrointestinal $(8,5 \%)$, próstata $(12,5 \%$; el $14 \%$ de los varones), vejiga $(8,5 \%)$, hígado $(3,7 \%)$, orofaringe $(3,7 \%)$ y sarcoma de Kaposi $(2,9 \%)$. La mortalidad varía significativamente 
según localización. La mortalidad fue mayor con los tumores pulmonares o hepáticos (un 79-85\% a los 5 años), mientras que la menor fue con los tumores de próstata (el 23\% a los 5 años) (DAC (Figura 15). A pesar de las limitaciones de este trabajo, inherentes a cualquier registro, entre ellas que los tratamientos se hicieron según criterio clínico del médico responsable, la mayor fortaleza es su tamaño muestral. El mensaje es que no todos los tumores tienen el mismo pronóstico tras el TxC. Esto tiene implicaciones en las actitudes que seguir, en concreto si se decide reducir la inmunosupresión con idea de retrasar la progresión tumoral, pero a expensas de aumentar el riesgo de rechazo y el consiguiente riesgo de muerte del paciente. Es necesario individualizar cada caso.

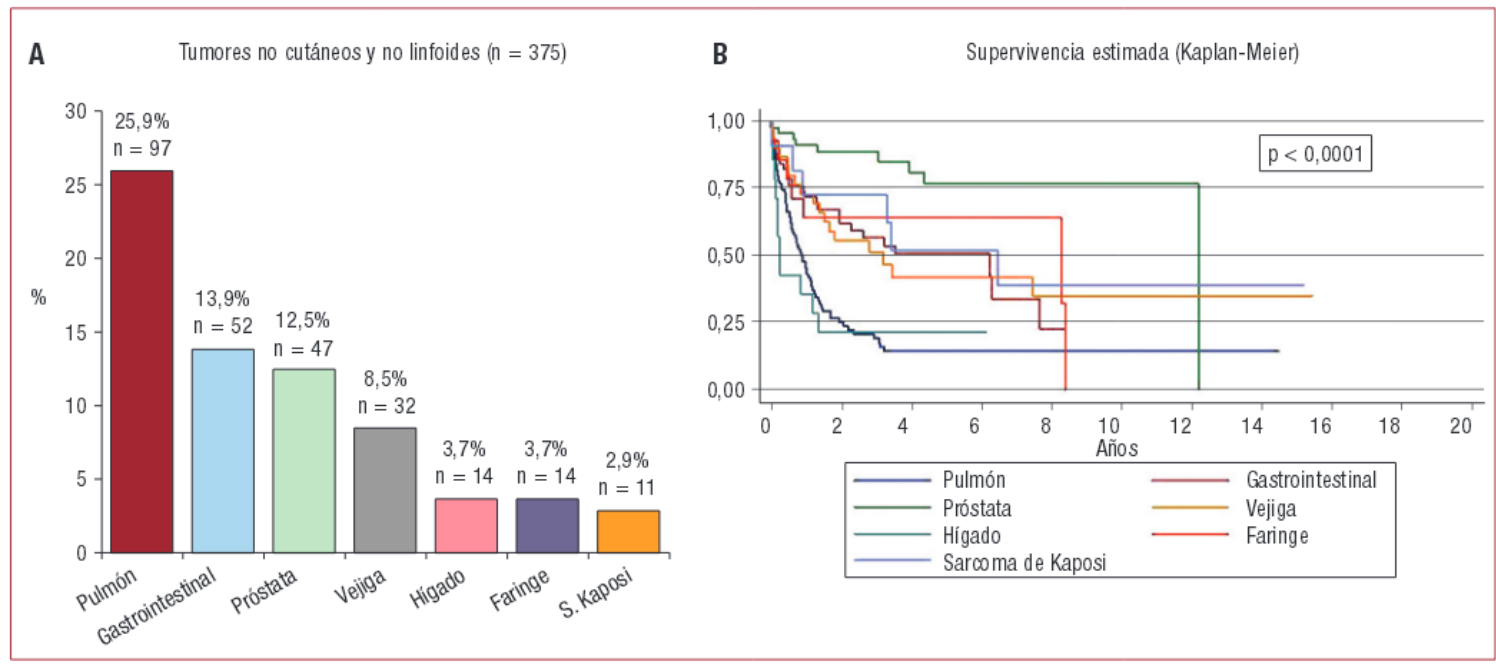

Figura 15. A: Tumores no cutaneos y no linfoides. B: Supervivencia por tipo de tumor (no cutaneo no linfoide). S. Kaposi: sarcoma de Kaposi.

\section{Neoplasias cutáneas postrasplante cardiaco}

Los tumores cutáneos son las neoplasias más frecuentes tras el TxC, más del $50 \%$ de todos los tumores. Se analizó en un grupo de 4.089 pacientes del RETPTC específicamente los tumores cutáneos diferentes del melanoma, y se apreció que el tumor más frecuente era el carcinoma de células escamosas, frente al carcinoma de células basales, que es el más frecuente en la población general. Se identificaron como factores de riesgo de ambos tipos de tumor la edad en el momento del TxC, el uso de terapia de inducción y la exposición solar. Con respecto a la inmunosupresión, se apreció que para el carcinoma de células escamosas la azatioprina aumentaba el riesgo relativo y el MMF tenía un efecto protector. Sin embargo, el riesgo relativo de carcinoma de células basales no se vio afectado por ningún fármaco inmunosupresor ${ }^{11}$.

\section{Linfomas postrasplante cardiaco}

Los linfomas postrasplante clásicamente se han asociado a la terapia de inducción y la infección por Herpesvirus $^{12,13}$. Dado que la terapia antiviral se utiliza cada vez más en el post-TxC y que los regímenes de inducción han variado con el tiempo, el RETPTC analizó en una cohorte de 3.393 pacientes si esto puede influir en la incidencia de linfomas ${ }^{14}$. Se apreció que las terapias de inducción con ATG u OKT3 incrementan el riesgo de linfomas o no dependiendo de si se utiliza profilaxis antiviral con aciclovir o ganciclovir en el post-TxC o no. La inducción con antirreceptores de la interleucina 2 no aumenta la incidencia de linfomas (Figura 16). Como consecuencia de esta observación, se aconsejó que, en caso de utilizar terapia de inducción agresiva (ATG, OKT3), se debería utilizar profilaxis antiviral para reducir el riesgo de linfomas. De hecho, en un análisis reciente del RETPTC sobre la incidencia de linfomas postTxC en España, se aprecia que esta ha disminuido a la mitad en la última década respecto a la década previa, y que en ello podrían haber influido las recomendaciones anteriores ${ }^{15}$. 


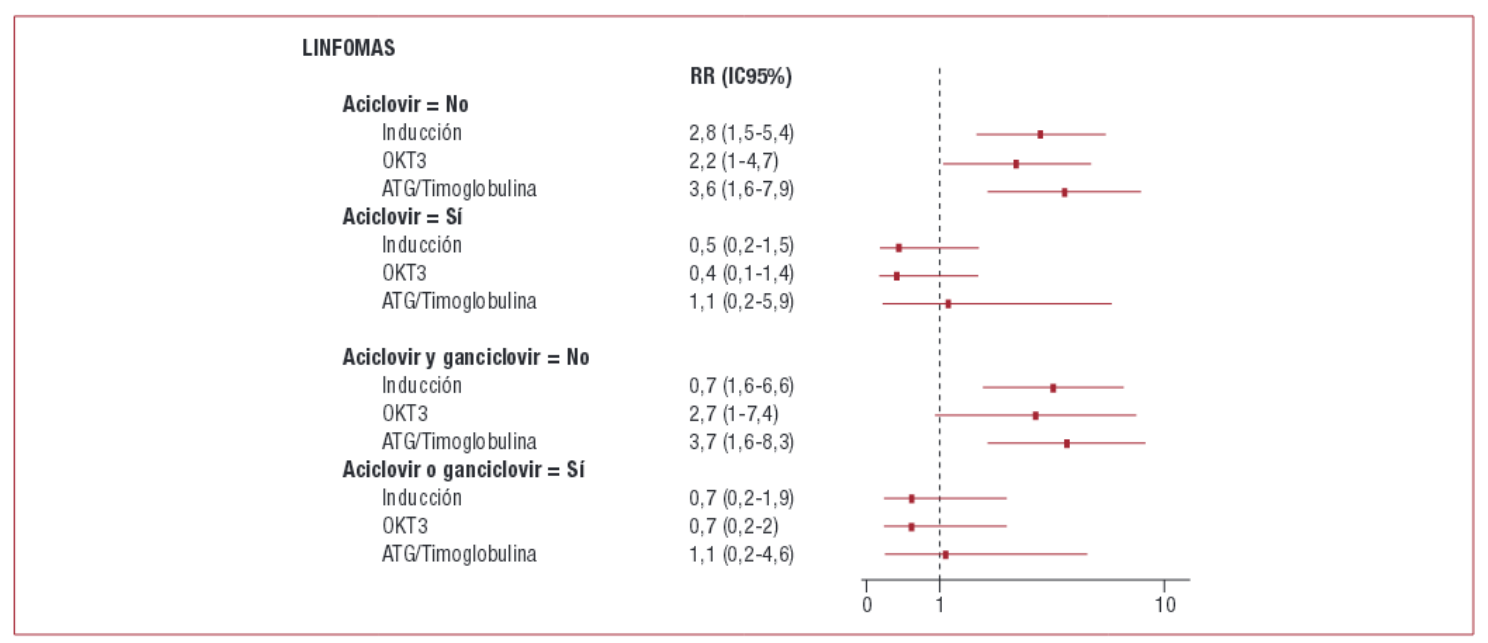

Figura 16. Efecto de la terapia de induccion (OKT3, ATG/timoglobulina) en el riesgo de linfomas, estratificado segun profilaxis antiviral (aciclovir, ganciclovir). ATG: globulina antitimocitaria.

\section{Cáncer de pulmón postrasplante cardiaco}

El cáncer de pulmón es el tumor sólido más frecuente tras el TxC, según los datos del RETPTC ${ }^{16}$. En un análisis específico sobre este tipo de cáncer, en el que se incluyeron 4.357 pacientes, hubo 102 con cáncer de pulmón (el 14\% de todos los pacientes con cáncer), generalmente carcinoma de células escamosas o adenocarcinoma, tras una media de 6,4 años post-TxC. Se apreció que la incidencia aumentaba con la edad en el momento del TxC, era mayor en varones que en mujeres y 4 veces mayor si había antecedentes de tabaquismo antes del TxC. La tasa de incidencia según la edad al diagnóstico era superior a la estimada para la población española según los datos de GLOBOCAN 2002; teniendo en cuenta también comparaciones con otros trabajos publicados, indica que el aumento en la incidencia no era solo explicable por la mayor prevalencia de fumadores entre los pacientes con TxC. El pronóstico de este tumor es muy malo, teniendo en cuenta además que el diagnóstico suele ser tardío; la mayoría de las veces, el tumor era ya inoperable en el momento del diagnóstico. Sin embargo, para los pacientes sometidos a cirugía curativa, la supervivencia a los 2 años era claramente superior (el 70 frente al $16 \%$ de los pacientes con tumor inoperable).

\section{Conclusiones}

Las neoplasias son una complicación grave y frecuente tras el TxC y una de las causas más importantes de mortalidad a largo plazo. El RETPTC, que recoge información de todos los pacientes que han recibido un trasplante en España y todos los tumores que se han desarrollado tras el $\mathrm{TxC}$, actualizado online continuamente, es una gran oportunidad para conocer mejor esta afección e identificar áreas de mejora.

Agradecimientos

Deseamos agradecer el apoyo prestado por Zulaika Grille en la coordinación del registro y a Francisco Arnal Monreal su asesoría como patólogo en el diseño de la base de datos.

Asimismo, agradecemos la participación de ODDS, S.L., empresa encargada de las labores de apoyo a la coordinación del registro y el análisis centralizado de datos (persona responsable: María Isolina Santiago).

\section{Financiación}

El RETPTC forma parte del Registro Nacional de Trasplante Cardiaco de la Sección de Insuficiencia Cardiaca y Trasplante de la Sociedad Española de Cardiología, y se financia en parte gracias a una ayuda de Novartis a esta Sección. Novartis no participa en la dirección y la gestión del Registro ni en modo alguno en la explotación de la información. La gran mayoría de los centros participantes en el Registro son miembros de la Red de Investigación Cardiovascular (RIC) del Instituto de Salud Carlos III. 


\title{
Conflicto de intereses
}

Ninguno.

\author{
Abreviaturas \\ MMF: micofenolato mofetilo \\ RETPTC: Registro Español de Tumores Postrasplante Cardiaco \\ TxC: trasplante cardiaco
}

\section{Anexo \\ Investigadores y colaboradores del Registro Español de Tumores Postrasplante Cardiaco.}

1. Hospital Universitario Puerta de Hierro, Madrid: Luis Alonso Pulpón (IP), Manuel Gómez Bueno, Javier Segovia Cubero.

2. Hospital Universitario 12 de Octubre, Madrid: Juan Delgado Jiménez (IP), Pilar Escribano, Miguel Ángel Gómez, Nuria Ochoa, Marta Paradinas, María José Ruiz Cano, María Vicente.

3. Hospital de la Santa Creu i Sant Pau, Barcelona: Vicens Brossa, Laura López, Sonia Mirabet (IP), Eulàlia RoigMinguell.

4. Hospital Universitario A Coruña, La Coruña: María Jesús Paniagua Martín, Eduardo Barge-Caballero, Maria G. Crespo Leiro (IP), Raquel Marzoa-Rivas, Zulaika Grille Cancela, Paula Blanco Canosa, Pilar Fariñas Garrido, Carmen Naya Leira.

5. Hospital General Universitario Gregorio Marañón, Madrid: Juan Fernández-Yáñez, Jesús Palomo, Iago Sousa Casasnovas (IP), Adolfo Villa Arranz

6. Hospital Universitari i Politècnic La Fe, Valencia: Luis Almenar Bonet (IP), Mónica Cebrián, Luis Martínez, Ignacio Sánchez-Lázaro.

7. Hospital Universitario Marqués de Valdecilla, Santander, Cantabria: Francisco González Vilchez (IP), José Antonio Vázquez de Prada.

8. Hospital Universitari de Bellvitge, L’Hospitalet de Llobregat, Barcelona: José González-Costello, Nicolás Manito Lorite (IP), Josep Roca.

9. Hospital Universitario Central de Asturias, Oviedo, Asturias: Beatriz Díaz Molina (IP), José Luis Lambert Rodríguez, María José Bernardo Rodríguez.

10. Clínica Universidad de Navarra, Pamplona, Navarra: Montserrat Lorente, Gregorio Rábago (IP).

11. Hospital Universitario Virgen del Rocío, Sevilla: Ernesto Lage Galle (IP), J. Manuel Sobrino.

12. Hospital Universitario Reina Sofía, Córdoba: José María Arizón del Prado (IP), Amador López-Granados.

13. Hospital Clínic i Provincial, Barcelona: Montserrat Cardona, $\mathbf{M}^{\mathrm{a}}$ Angeles Castel, Félix Pérez Villa (IP), Marta Farrero.

14. Hospital Universitario Miguel Servet, Zaragoza: Teresa Blasco Peiró (IP), Marisa Sanz Julve.

15. Hospital Clínico Universitario de Valladolid, Valladolid: Luis de la Fuente Galán (IP), Javier López Díaz.

16. Hospital Universitario Virgen de la Arrixaca, El Palmar, Murcia: Domingo Pascual Figal (IP), Iris Garrido Bravo.

17. Instituto Universitario de Ciencias de la Salud de la Universidad de A Coruña e Instituto de Investigación Biomédica de A Coruña (INIBIC), A Coruña: Javier Muñiz.

\section{Bibliografía}

1.Lund LH, Edwards LB, Kucheryavaya AY, Benden C, Christie JD, Dipchand AI, et al. The Registry of the International Society for Heart and Lung Transplantation: Thirty-first Official Adult Heart Transplant Report-2014; Focus Theme: Retransplantation. J Heart Lung Transplant. 2014; 33:996-1008.

2.Nair N, Gongora E, Mehra MR. Long-term immunosuppression and malignancy in thoracic transplantation: where is the balance? J Heart Lung Transplant. 2014; 33:461-7.

3.Penn I. Cancers of the anogenital region in renal transplant recipients Analysis of 65 cases. Cancer. 1986; 58:611-6.

4.Penn I. Incidence and treatment of neoplasia after transplantation. J Heart Lung Transplant. 1993; 12 (6 Pt 2):S32836.

5.Penn I. Post-transplant malignancy: the role of immunosuppression. Drug Saf. 2000; 23:101-13. 
6.Roithmaier S, Haydon AM, Loi S, Esmore D, Griffiths A, Bergin P, et al. Incidence of malignancies in heart and/or lung transplant recipients: a single-institution experience. J Heart Lung Transplant. 2007; 26:845-9.

7.Gao SZ, Chaparro SV, Perlroth M, Montoya JG, Miller JL, DiMiceli S, et al. Post-transplantation lymphoproliferative disease in heart and heart-lung transplant recipients: 30-year experience at Stanford University. J Heart Lung Transplant. 2003; 22:505-14.

8.El-Hamamsy I, Stevens LM, Carrier M, Pelletier G, White M, Tremblay F, et al. Incidence and prognosis of cancer following heart transplantation using RATG induction therapy. Transpl Int. 2005; 18:1280-5.

9.Crespo-Leiro MG, Alonso-Pulpon L, Vazquez de Prada JA, Almenar L, Arizon JM, Brossa V, et al. Malignancy after heart transplantation: incidence prognosis risk factors. Am J. Transplant. 2008; 8:1031-9.

10.Crespo-Leiro MG, Alonso-Pulpon LA, Villa-Arranz A, Brossa-Loidi V, Almenar-Bonet L, Gonzalez-Vilchez F, et al. The prognosis of noncutaneous, nonlymphomatous malignancy after heart transplantation: data from the Spanish Post-Heart Transplant Tumour Registry. Transplant Proc. 2010; 42:3011-3.

11. Molina BD, Leiro MG, Pulpon LA, Mirabet S, Yanez JF, Bonet LA, et al. Incidence and risk factors for nonmelanoma skin cancer after heart transplantation. Transplant Proc. 2010; 42:3001-5.

12. Newell KA, Alonso EM, Whitington PF, Bruce DS, Millis JM, Piper JB, et al. Posttransplant lymphoproliferative disease in pediatric liver transplantation Interplay between primary Epstein-Barr virus infection and immunosuppression. Transplantation. 1996; 62:370-5.

13. Swinnen L, Costanzo-Nordin M, Fisher S, O'Sullivan E, Johnson M, Heroux A, et al. Increased incidence of lymphoproliferative disorder after inmunosuppression with the monoclonal antibody OKT3 in cardiac transplant recipients. N Engl J Med. 1990; 323:1723-8.

14.Crespo-Leiro MG, Alonso-Pulpon L, Arizon JM, Almenar L, Delgado JF, Palomo J, et al. Influence of induction therapy, immunosuppressive regimen and anti-viral prophylaxis on development of lymphomas after heart transplantation: data from the Spanish Post-Heart Transplant Tumour Registry. J Heart Lung Transplant. 2007; 26:1105-9.

15.Crespo-Leiro MG, Delgado-Jimenez J, Lopez L, Alonso-Pulpon L, Gonzalez-Vilchez F, Almenar-Bonet L, et al. The falling incidence of hematologic cancer after heart transplantation. Clin Transplant. 2014; 28:1142-7.

16. Crespo-Leiro MG, Villa-Arranz A, Manito-Lorite N, Paniagua-Martin MJ, Rabago G, Almenar-Bonet L, et al. Lung cancer after heart transplantation: results from a large multicenter registry. Am J Transplant. 2011; 11:103540 . 